\title{
Reabilitação estética funcional com resina composta: relato de caso
}

\author{
Functional aesthetic rehabilitation with composite resin: case report
}

\author{
Rehabilitación estética funcional con resina compuesta: reporte de caso
}

\begin{abstract}
Heloisa França de Araujo ${ }^{1 *}$, Héberte de Santana Arruda ${ }^{1}$, Sérgio Murilo Cordeiro de Melo Filho1, Beatriz Borba Barros Bernardo ${ }^{1}$, Mariana Alves Lemos², Amanda Maciel do Prado1, Zilda Betânia Barbosa Medeiros de Farias ${ }^{1}$, Maria Cristina Valença de Oliveira ${ }^{1}$, Eduardo Borges da Costa Leite $^{3}$, Marcos Antonio Japiassú Resende Montes'.
\end{abstract}

\section{RESUMO}

Objetivo: Descrever reabilitação estética anterossuperior através de facetas diretas em resina composta. Relato de Caso: Paciente procurou a clínica odontológica, pois estava insatisfeita com seu sorriso. Ao exame clínico foi constatada perda de dimensão vertical, hábitos parafuncionais de bruxismo de vigília e restaurações infiltradas, dentre outros achados. Inicialmente, foi realizada uma profilaxia com pasta à base de pedra-pomes e água, em escova de Robinson, de todas as superfícies dentais; em seguida, após a confecção de uma placa miorrelaxante de acetato, visando controlar o bruxismo, foram realizadas restaurações adesivas nos dentes posteriores com o intuito de aumentar a dimensão vertical de oclusão. Sendo assim, os elementos fraturados que apresentavam férula compatível, optou-se por realizar a reconstrução com pino de fibra de vidro em virtude de eliminar a etapa laboratorial. Posterior a isso foi realizada uma sessão de clareamento de consultório e pôr fim a reabilitação através de facetas diretas em resina composta. Considerações finais: No tratamento reabilitador estético se fazem de extrema importância a destreza e competência do cirurgião-dentista quanto às técnicas e conhecimentos empregados no caso, visando à satisfação e segurança do paciente e êxito do tratamento realizado.

Palavras-chave: Facetas dentárias, Bruxismo, Clareamento dental, Resinas compostas, Reabilitação.

\begin{abstract}
Objective: To describe a case report of anterosuperior aesthetic rehabilitation through direct facets in composite resin. Case Report: The patient sought the dental clinic because he was dissatisfied with your smile. The clinical examination revealed a loss of vertical dimension, parafunctional waking bruxism and infiltrated restorations, among other findings. Initially, prophylaxis was performed with pumice paste and water, using a Robinson brush, of all dental surfaces; then, after making an acetate myorelaxant plate, aiming to control bruxism, adhesive restorations were performed on the posterior teeth in order to increase the vertical dimension of occlusion. Thus, the fractured elements that had a compatible template, it was decided to perform the reconstruction with a fiberglass pin in order to eliminate the laboratory stage. After that, an office whitening session was held and an end to rehabilitation through direct facets in composite resin. Final considerations: In the aesthetic rehabilitation treatment, extreme importance the skill and competence of the dentist regarding the techniques and knowledge employed in the case, aiming at patient satisfaction and safety and the success of the treatment performed.
\end{abstract}

Keywords: Dental veneers, Bruxism, Tooth bleaching, Composite resins, Rehabilitation.

\footnotetext{
1 Universidade de Pernambuco (FOP/UPE), Camaragibe - PE. *E-mail: heloisaraujo24@gmail.com.

2 Instituto de Medicina Integral Professor Fernando Figueira (IMIP), Recife - PE.

3 Universidade Federal de Pernambuco (UFPE), Recife - PE.
} 


\section{RESUMEN}

Objetivo: Describir la rehabilitación estética superior anterior a través de facetas directas en resina compuesta. Informe del Caso: El paciente buscó la clínica dental porque estaba insatisfecho con tu sonrisa. El examen clínico reveló una pérdida de dimensión vertical, bruxismo parafuncional de vigilia y restauraciones infiltradas, entre otros hallazgos. Inicialmente, la profilaxis se realizó con pasta de piedra pómez y agua, utilizando un cepillo Robinson, de todossuperficies dentales; luego, después de hacer una placa de acetato miorrelajante, con el objetivo de controlar bruxismo, se realizaron restauraciones adhesivas en los dientes posteriores para aumentar la dimensión vertical de la oclusión. Por lo tanto, los elementos fracturados que tenían una plantilla compatible, se decidió realizar la reconstrucción con un alfiler de fibra de vidrio para eliminar la etapa de laboratorio. Después de eso, se realizó una sesión de blanqueamiento en la oficina y el final de la rehabilitación a través de facetas directas en resina compuesta. Consideraciones finales: En el tratamiento de rehabilitación estética, extrema Importancia de la habilidad y competencia del dentista con respecto a las técnicas y el conocimiento empleado en el caso, con el objetivo de la satisfacción del paciente y la seguridad y el éxito del tratamiento realizado.

Palabras clave: Coronas con frente estético, Bruxismo, Blanqueamiento de dientes, Resinas compuestas, Rehabilitación.

\section{INTRODUÇÃO}

Por conceito, estética é a apreciação de beleza. Apreciar o belo está relacionado à sensação de prazer diante da visualização de um objeto, pessoa ou som; sendo assim, o conceito de beleza é próprio de cada indivíduo, estabelecido a partir de valores individuais e varia em diferentes momentos da história (GIDDON DB, 1978; REIS SAB, et al., 2006). O apelo e a demanda pela estética crescem cada vez mais na nossa sociedade. No meio odontológico ela se apresenta como um segmento bastante representativo onde, cada vez mais, os pacientes exigem dentes com maior naturalidade e harmonia com o rosto.

A procura por tratamentos estéticos nos consultórios odontológicos é crescente devido principalmente a atender padrões subliminarmente impostos pela sociedade. Grande parte dos pacientes que buscam por tratamentos visam não apenas saúde ou restabelecimento de função, mas também intervenções que influenciem em sua autoimagem, seja por vaidade ou aceitação social (PEREIRA DA, et al., 2016).

Desta forma, procedimentos estéticos como correção de imperfeições e clareamentos tem sido cada vez mais recorrentes buscando uma harmonia no sorriso e o bem-estar do paciente, elevando assim sua autoestima (SILVA LA, et al., 2006)

O aumento da demanda pela odontologia estética e cosmética tem redefinido o mercado odontológico com a pesquisa e o desenvolvimento de novos materiais que procuram aliar propriedades mecânicas satisfatórias a um bom comportamento estético. Sendo assim, novas técnicas restauradoras, melhoria dos sistemas adesivos e mecanismos de polimerização, bem como melhores propriedades físicas e mecânicas das resinas compostas vêm surgindo e, dessa forma, permite ao cirurgião-dentista executar uma abordagem mais conservadora em seus procedimentos (LEITE EBC, et al., 2016; SILVA AM, et al., 2018; OLIVEIRA AS, et al., 2019).

Entretanto, há situações onde ser conservador não é a melhor escolha, se fazendo necessário recorrer a intervenções mais invasivas tais como procedimentos indiretos e/ou intervenções protéticas (HEINTZE SD, et al., 2015; GRESNIGT MM, et al., 2013; MANHART J, et al., 2004).

Leite EBC, et al. (2016), afirmam que as técnicas clareadoras são comumente associadas às restauradoras a fim de proporcionar um trabalho mais harmônico aos pacientes. Sendo assim, as facetas diretas em resina composta surgem como uma boa alternativa para contornar detalhes que influenciam diretamente na harmonia do sorriso tais como o escurecimento dentário, restaurações extensas deficientes, 
alterações de forma, cor e textura em dentes vitais ou não vitais. Atualmente tornou-se possível agregar o mínimo desgaste da estrutura dentária à adesividade, resistência, durabilidade e agilidade na confecção, acompanhada de resultados previsíveis que satisfaçam as expectativas do paciente.

As facetas diretas em resina composta têm como vantagens serem uma técnica rápida, segura e eficaz; terem menor custo em relação às cerâmicas; dispensam etapas de laboratório e não requerem provisório e nem moldagem (SANTOS JS, 2016). Vieira APSB, et al. (2018), cita também a preservação da estrutura dental, reforço do remanescente dentário e obtenção de resultados de excelência em virtude da variedade de compósitos com diferentes graus de opacidade e translucidez.

É importante salientar que o planejamento é uma etapa crucial nos tratamentos odontológicos reabilitadores. A experiência do cirurgião-dentista aliada ao conhecimento técnico-científico e às suas habilidades psicomotoras determinarão o caminho a ser seguido, pois, na realidade, existem várias possibilidades para atingir-se o resultado final. Sendo assim, a decisão terapêutica a ser tomada deve considerar as necessidades estéticas frente às alternativas disponíveis, bem como a presença de cárie, o grau de destruição dental e a qualidade das restaurações existentes.

O plano de tratamento também pode ser influenciado por preferências, motivação, saúde sistêmica, estado emocional, além de poder ser modificado em virtude do conhecimento, experiência e treinamento do profissional, suporte laboratorial, relacionamento profissional-paciente e integração com as outras especialidades necessárias à viabilização do tratamento restaurador (VIEIRA APSB, et al., 2018; MOREIRA EJR, et al., 2018).

A evolução dos materiais restauradores, sem dúvida, trouxe consigo uma diversidade de sistemas de resinas compostas, permitindo o uso de diferentes combinações de cores, saturações, translucidez e opacidades, sendo que detalhes específicos existentes na dentição natural podem ser reproduzidos, mantendo um adequado comportamento com relação à estabilidade de cor e resistência ao desgaste (PONTONS-MELO JC, 2013). É imprescindível que o cirurgião-dentista esteja atualizado frente essa evolução visando planejar e executar o melhor tratamento para o paciente. Sendo assim, o objetivo desse artigo é discorrer, através de um relato de caso, sobre uma reabilitação estética anterossuperior em resina composta.

\section{RELATO DE CASO}

Paciente de 52 anos, do sexo feminino, procurou a Clínica do Curso de Especialização em Dentística, pois se aproximava a formatura da filha e a paciente encontrava-se insatisfeita com o seu sorriso, sentia vergonha da condição em que os dentes se encontravam; gostaria de voltar a sorrir. Durante a avaliação, constatou-se a presença de restaurações infiltradas, elementos dentários fraturados, diastemas, presença de hábito deletério (apertamento dos maxilares), bem como a perda de elementos posteriores levando a uma perda de dimensão vertical, que certamente contribuiu para o agravamento da situação (Figuras $1 \mathrm{~A}$ e 1B).

Figuras 1A e 1B - Registro fotográfico da condição clínica inicial da paciente.

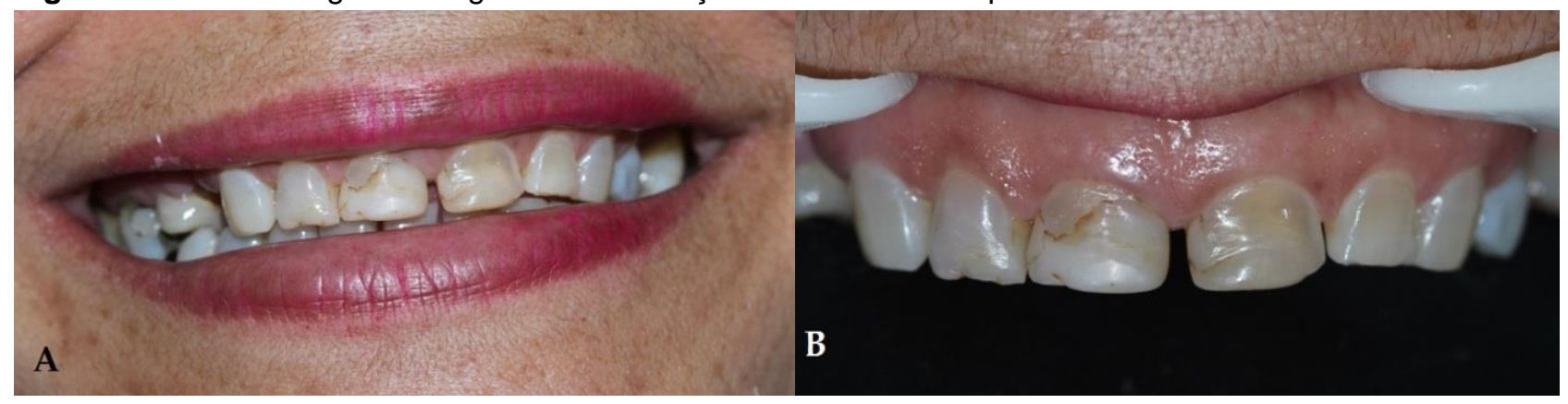

Fonte: Leite EBC, et al., 2019. 
Após realização de exame clínico, radiográfico, fotográfico e de confecções de modelos de estudo, foi elaborado um plano de tratamento que se consistiu em ganhar alguns milímetros em dimensão vertical através da reconstrução dos elementos posteriores fraturados bem como a utilização uma placa miorrelaxante de acetato (2mm) (Figura 3), sessões de clareamento de consultório, para um resultado mais eficaz e restaurações adesivas nos dentes anteriores, com o intuito de fechamento de diastemas e melhorias na proporção dental.

Figura 3 - Modelo de gesso para confecção da placa miorrelaxante.

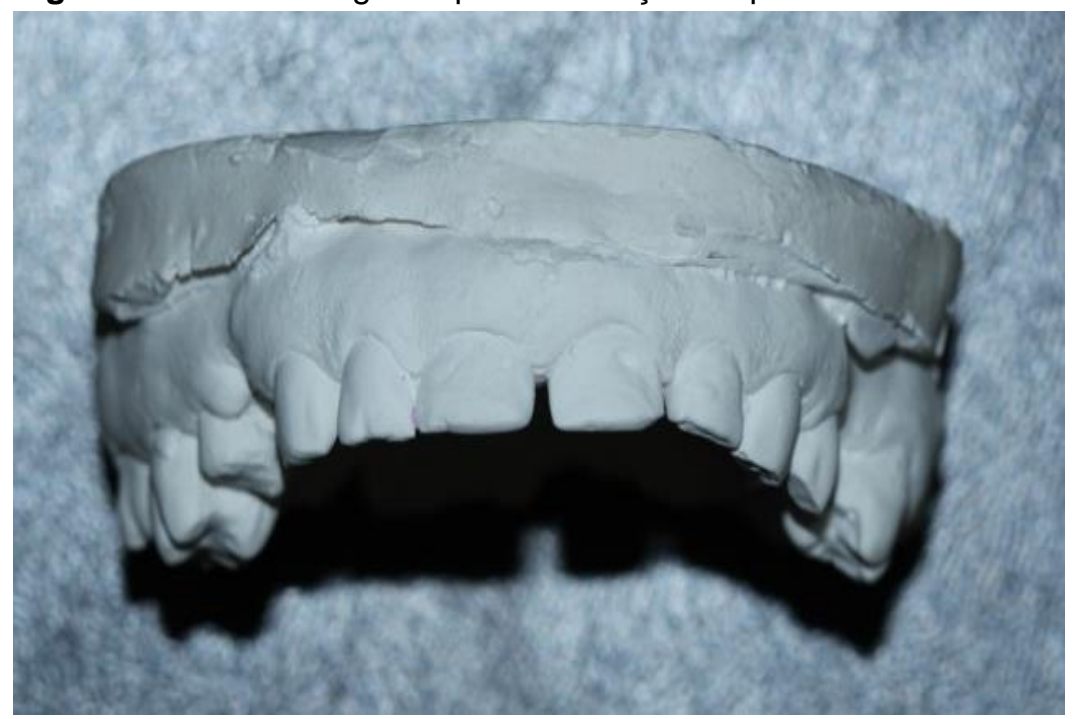

Fonte: Leite EBC, et al., 2019.

Inicialmente, foi realizada uma profilaxia com pasta à base de pedra-pomes e água, em escova de Robinson, de todas as superfícies dentais. Logo em seguida utilizou-se o modelo de estudo para confecção da placa miorrelaxante, que, preferivelmente, foi feita em acetato em virtude de ser um material mais resistente, objetivando uma maior estabilização da oclusão.

Após essa etapa, foram realizadas restaurações adesivas nos dentes posteriores visando aumentar a dimensão vertical de oclusão. Sendo assim, os elementos fraturados que apresentavam férula compatível, optou-se por realizar a reconstrução com pino de fibra de vidro em virtude de eliminar a etapa laboratorial, já que se aproximava a data da formatura de sua filha.

Realizou-se a desobturação parcial do conduto com brocas Largo compatíveis com a anatomia do conduto radicular, deixando apenas $4 \mathrm{~mm}$ de obturação, condizentes com a literatura. Após o condicionamento do remanescente dentário, do pino e das etapas adesivas, deu-se a cimentação do mesmo utilizando o cimento resinoso dual Allcem Core, que também foi utilizado para a construção do munhão em razão das suas propriedades tixotrópicas.

As reconstruções dos elementos foram realizadas através de resina filtek Z350 de dentina, corpo e esmalte. Os demais elementos posteriores receberam restaurações adesivas convencionais, quando necessário. A princípio a paciente queixou-se muito sobre a nova condição. $O$ aumento da dimensão vertical por meio das restaurações lhe causou estranheza, sensação de algo errado. Porém, após receber as devidas orientações, foi liberada com a orientação de utilizar a placa miorrelaxante ao dormir.

Na sessão seguinte, observou-se a presença de áreas de retração gengival, que receberam tratamento prévio antes da realização do procedimento clareador. As áreas de dentina expostas, sem perda de tecido dental, receberam condicionamento ácido com ácido fosfórico, por trinta segundos, e aplicação de duas camadas de adesivo, sob isolamento relativo com afastador labial, roletes de algodão e sugador. As regiões que apresentavam alguma cavitação foram devidamente restauradas com ionômero de vidro modificado por resina. 
Posteriormente, deu-se a aplicação do agente clareador (Whiteness HP 35\%) o qual agiu na estrutura dentária por 40 minutos. Ao final da sessão clínica, o gel clareador foi removido utilizando uma cânula aspiradora e os dentes foram lavados com água e, em seguida, foi aplicado o agente dessensibilizante (Nano P) na região cervical dos elementos. Foi realizada apenas uma sessão clínica, obtendo-se uma coloração final satisfatória a paciente.

Após uma semana do término da sessão de clareamento fez-se o registro da cor da resina composta dando-se início, após a realização do isolamento, ao tratamento restaurador. Inicialmente realizou-se o condicionamento ácido com ácido fosfórico 37\% (CONDAC), por um minuto, seguido da aplicação do sistema adesivo (Single Bond), conforme manda o fabricante. Após essa etapa, teve início as restaurações adesivas, as quais foram realizadas com resina composta (Z350, 3M ESPE) por meio da técnica de estratificação (Figura 4).

Figura 4 - Registro fotográfico após a realização do clareamento e substituições das restaurações.

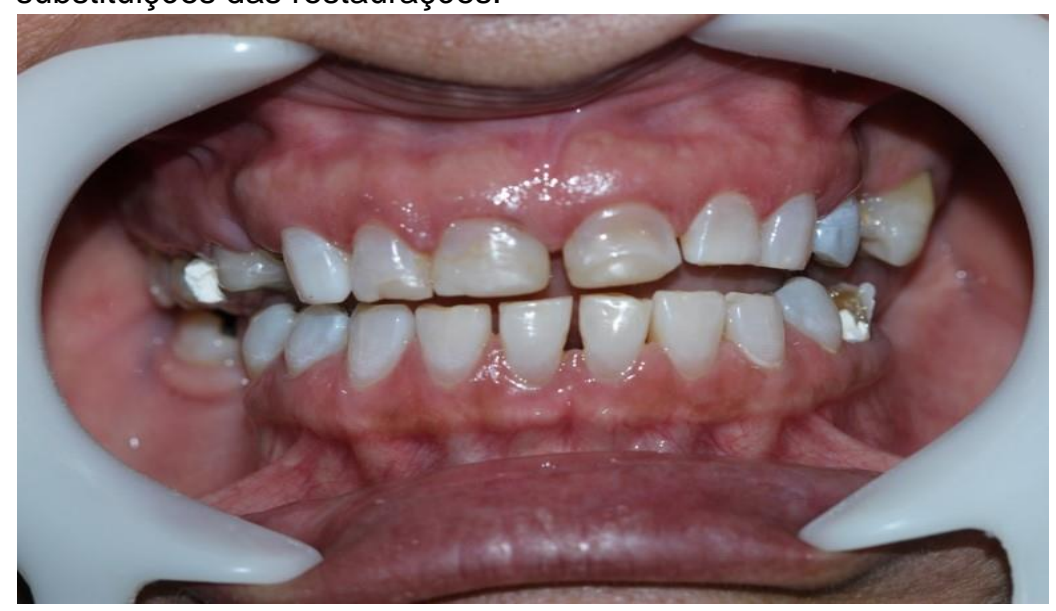

Fonte: Leite EBC, et al., 2019.

Com o término dessa etapa, foi realizado o acabamento inicial removendo os pequenos excessos combisturi, discos de lixa e tiras abrasivas. Na sessão seguinte, após os dentes retomarem sua hidratação natural, retomou-se o acabamento e polimento final (Figuras 5A e 5B).

Figuras 5A e 5B - Registro fotográfico ao final do tratamento.

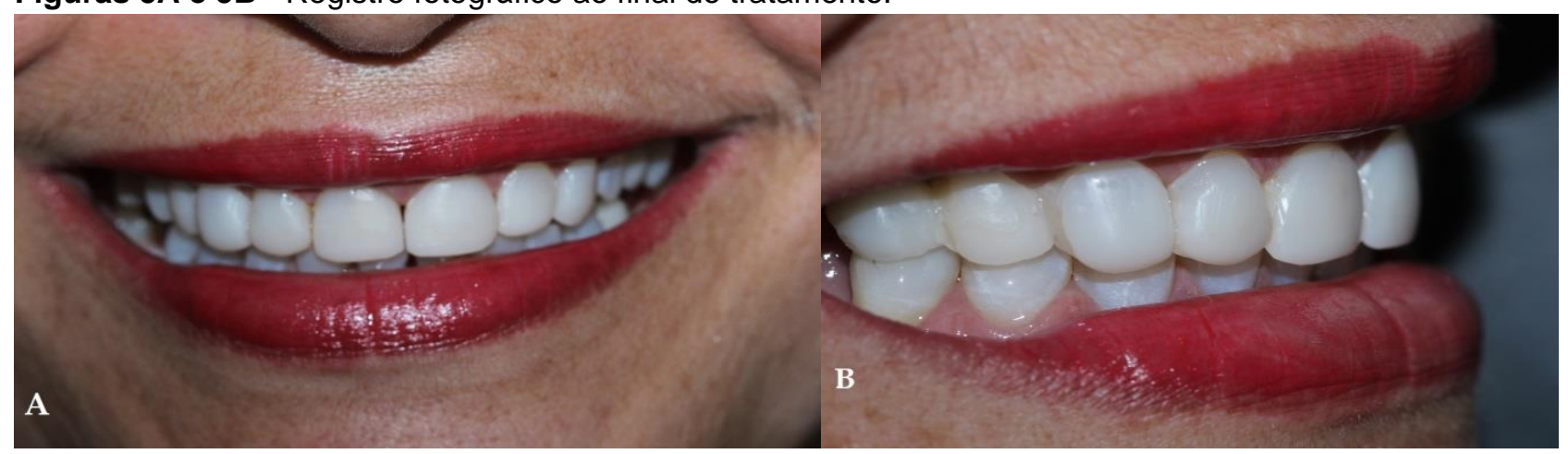

Fonte: Leite EBC, et al., 2019.

\section{DISCUSSÃO}

Segundo Silva LA, et al. (2006), o tratamento estético deve apresentar uma abordagem multidisciplinar, utilizando de vários recursos, técnicas e materiais restauradores para a melhoria do sorriso. Sendo assim, partindo de procedimentos adesivos, técnicas de clareamento dentário e remodelação cosmética, juntamente com conhecimentos de Ortodontia, Periodontia e Oclusão, é possível modificar forma, cor, tamanho e posição dos dentes. 
O mesmo é relatado por Caldo-Teixeira AS, et al. (2009), quando defendem que devido às diversas etiologias, diferentes condutas podem ser realizadas, podendo envolver muitas vezes, mais de uma especialidade. Desta forma, torna-se imprescindível o correto diagnóstico e plano de tratamento. $O$ conhecimento científico adequado aliado às diretrizes que regem a odontologia estética confere ao profissional grandes possibilidades de devolver forma, cor e textura ao dente afetado.

O recontorno cosmético com resinas compostas é a técnica mais simples de restauração direta, menos invasiva e geralmente requer apenas um condicionamento ácido adequado, não necessitando de nenhum tipo de preparo prévio no substrato de destino pois a adesão ao esmalte é um procedimento amplamente aceito e comprovado.

Sendo baseada na aposição de resinas compostas para promover o recontorno cosmético, essa técnica é indicada nos casos em que não existam alterações severas de cor e/ou quando o posicionamento não requer nenhuma correção por meio de desgaste, podendo também ser empregada para o fechamento de diastemas e restaurações de dentes conóides, visando sempre ao reposicionamento adequado do dente no arco (HIGASHI C, et al., 2006).

Também se faz necessário levar em consideração o conceito de oclusão ideal o qual reza que a mesma apresenta muitas variações, de acordo com o objetivo para o qual a análise oclusal está sendo realizada. A dimensão vertical de oclusão é caracterizada não apenas pela distância vertical entre a mandíbula e a maxila quando os dentes estão em contato, mas por um equilíbrio no relacionamento maxilomandibular que envolve alterações neuromusculares, morfológicas e funcionais que refletem na estética, mas principalmente nos processos de mastigação, deglutição e fala de cada indivíduo. Caso a mesma não seja restabelecida corretamente, poderá acarretar em danos a todo sistema estomatognático.

Dessa forma o restabelecimento da mesma é de fundamental importância do ponto de vista estético para recuperação da altura do terço inferior da face e do equilíbrio facial e do ponto de vista funcional, para criar um espaço que permita a reconstrução oclusal, mantendo características adequadas de oclusão.

Nesses casos, torna-se imperativa a análise dessa dimensão, que deve ser feita mediante uma avaliação da estabilidade oclusal, do histórico do desgaste, de testes fonéticos, da distância interarcos e da aparência facial (PEGORARO LF, et al., 2013).

Diante da evolução dos materiais, novas propriedades físicas e mecânicas vêm surgindo e, assim, permite ao cirurgião-dentista executar uma abordagem mais conservadora em seus procedimentos (OLIVEIRA AS, et al., 2019; SILVA AM, et al., 2018).

No presente relato é notória a presença de diastemas, que podem ser trabalhados tanto por meios ortodônticos quanto por procedimentos restauradores. Neste caso, a reabilitação em facetas diretas de resina composta visa aumentar as dimensões dentárias por intermédio de técnicas e materiais específicos que deverão ser escolhidos levando em consideração os conhecimentos técnico-científicos do profissional assim como as condições econômicas, físicas e fisiológicas do paciente (PRABHU R, et al., 2015).

Segundo Coelho-de-Souza FH, et al. (2015), as facetas diretas em resina composta são a primeira escolha nos procedimentos que necessitam um mínimo desgaste da estrutura dental, dessa forma, diferindo dos procedimentos indiretos que, além de necessitarem de um desgaste maior da estrutura dentária, tem um custo mais elevado. Esse procedimento vem se tornando uma excelente alternativa de tratamento para problemas estéticos em dentes anteriores.

A aplicação em uma única sessão do material restaurador definitivo com o mínimo de desgaste ou algumas vezes sem nenhum desgaste da superfície dentária é uma das vantagens dessa técnica (KORKUT B, 2018).

As facetas apresentam como características preparos menos invasivos e mais rápido quando comparados com as coroas totais protéticas e possibilita a realização de preparos supra gengivais o que leva uma melhor resposta do tecido periodontal (MENDES WB, et al., 2004). 
Ademais, elas não necessitam de etapas laboratoriais, isso diminui o seu custo e torna o tratamento mais rápido, apresentam maior facilidade de reparação e, além disso, permite sua utilização por pacientes que apresentam parafunção uma vez que as resinas compostas se adaptam melhor a essa condição, pois possuem baixa probabilidade de desgastar os dentes antagonistas (CUNHA ARFMD, 2013).

Mondelli J, et al. (2003) afirmam que a remodelação cosmética dos dentes naturais, além dos benefícios de aparência, melhora também, em algumas situações, a função, higiene e o periodonto.Em contrapartida, as resinas compostas apresentam algumas desvantagens, como: dificuldade na obtenção de pontos de contato, contração de polimerização que leva a ocorrência de fendas marginais e micro-infiltração, baixa resistência ao desgaste e dificuldade de mascarar substratos muito escurecidos quando comparadas, por exemplo à porcelana (CUNHA ARFMD, 2013).

Os materiais disponíveis na atualidade, com o advento da nanotecnologia, proporcionam ao profissional excelentes propriedades de textura, maleabilidade, módulo de elasticidade, adesividade, o que interfere diretamente no resultado final, porém, ainda sim têm limitações que lhe são intrínsecas.

Uma das formas de minimizar essas desvantagens da resina composta quanto à instabilidade da cor e a infiltração marginal é através da realização de um acabamento e polimento bem feito nas restaurações com vista a garantir uma maior longevidade e, além disso, fazer o acompanhamento do paciente regularmente (KORKUT B, et al., 2016).

A anamnese e o diagnóstico precisos são de extrema importância para a escolha e indicação do material e técnica a serem utilizados. É imprescindível que o cirurgião-dentista tenha conhecimento das propriedades de cada material e dessa forma esteja apto a selecionar o melhor para cada caso, levando em consideração que todos os materiais e técnicas têm vantagens, desvantagens, indicações e contraindicações e não existe o melhor entre eles, mas sim o mais indicado para cada caso (CUNHA ARFMD, 2013).

Para se obter sucesso no tratamento reabilitador estético, o cirurgião-dentista deve considerar todas as nuances que o paciente apresenta, realizando um diagnóstico e plano de tratamento singularizado para cada caso, optando pelas melhores técnicas e conhecimentos científicos a fim de proporcionar ao paciente o melhor resultado possível, com segurança e êxito.

O tratamento proposto no caso relatado foi planejado e executado visando as necessidades específicas da paciente, fato este que justifica o sucesso obtido e a satisfação da paciente. O restabelecimento da DVO foi fundamental, já que sem ela não teria sido possível criar os espaços para reconstrução dos elementos e assim devolver a harmonia ao sorriso. Com o advento das nanotecnologias e aumento da excelência dos materiais dentários disponíveis na Odontologia é possível realizar um tratamento satisfatório em um curto espaço de tempo e com procedimentos minimamente invasivos.

\section{REFERÊNCIAS}

1. ARRUDA HS, JÚNIOR AAG, LEITE EBC. Reabilitação estética anterior: do clareamento à harmonização do sorriso. Odontol. Clín.-Cient., 2016; 15(2): 83-90

2. CALDO-TEIXEIRA AS, et al. Fechamento de diastemas com restaurações diretas de resina composta: relato de caso clínico. Revista Gestão \& Saúde, 2009; 1(3): 33-38

3. COELHO-DE-SOUZA FH, et al. Direct anterior composite veneers in vital and non-vital teeth: A retrospective clinical evaluation. Journal of Dentistry, 2015; 43(11): 1330-1336

4. CUNHA ARFMD. Facetas de Porcelana VS Facetas de Resina Composta. Dissertação (Mestrado Integrado em Medicina Dentária). Universidade do Porto, Porto, 2013; $80 \mathrm{p}$

5. GIDDON DB. Aplicações ortodônticas de estudos psicológicos e perceptuais da estética facial. In: LEVIN, El. Dental esthetics and the golden proportion. The Journal of Prosthetic Dentistry, 1978; 40(3): 244-252.

6. GRESNIGT MM, et al. Randomized clinical trial of indirect resin composite and ceramic veneers: up to 3-year followup. J Adhes Dent, 2013; 15(2): 181-190 
7. HEINTZE SD, et al. Clinical effectiveness of direct anterior restorations-a meta-analysis. Dental materials, 2015; $31(5): 481-495$

8. HIGASHI C,et al. Planejamento estético em dentes anteriores. In: MIYASHITA E, MELLO AT. Odontologia estética planejamento e técnica. São Paulo: Artes Médicas, 2006. cap. 7, p. 139-154

9. KORKUT B, et al. Direct Midline Diastema Closure with Composite Layering Technique: A One-Year Follow-Up. Case Reports in Dentistry, 2016; 2016: 1-5.

10. KORKUT B. Smile makeover with direct composite veneers: A two-year follow-up report. J Dent Res DentClinDentProspect, 2018; 12(2): 146-151

11. MANHART J. et al. Review of the clinical survival of direct and indirect restorations in posterior teeth of the permanent dentition. OPERATIVE DENTISTRY-UNIVERSITY OF WASHINGTON, 2004; 29: 481-508

12. MENDES WB, et al. Facetas laminadas - Cerâmica e Resina: Aspectos Clínicos. In: Livro do Ano da Clínica Odontológica Brasileira.São Paulo: Artes Médicas: 2004.cap. 2, p. 27-59

13. MONDELLI J. Estética e Cosmética em Clínica Integrada Restauradora. São Paulo: Santos, $2003 ; 476$

14. MOREIRA EJR, et al. Harmonização estética do sorriso com facetas diretas em resina composta: relato de caso. ScilnvestDent, 2018; 23(1): 22-27

15. OLIVEIRA AS, et al. Mascaramento de dentes escurecidos utilizando restaurações diretas: relato de caso. Revista Diálogos Acadêmicos, 2019; 8(2): 35-41

16. PEGORARO LF. Prótese fixa: bases para o planejamento em reabilitação oral. 2ª ed. São Paulo: Artes Médicas, 2013; 487p.

17. PEREIRA DA, et al. Reabilitação estética do sorriso por meio de procedimento restaurador direto com resina composta nanoparticulada: relato de caso. Revista Odontológica do Brasil Central, 2016; 25(72): 54-58.

18. PONTONS-MELO JC. Harmonização do sorriso por meio de procedimentos minimamente invasivos. FGM News, 2013; 132-137

19. PRABHU R, et al. Clinical evaluation of direct composite restoration done for midline diastema closure - long-term study. Journal of Pharmacy \&BioAllied Sciences, 2015; 7(6): 559-562.

20. REIS SAB, et al. Análise Facial Subjetiva. Rev. Dent. Press Ortodon. Ortop. Facial, 2006; 11(5): $159-172$.

21. SANTOS JS. Abordagem na seleção de materiais para facetas diretas de resina composta na resolução estética em dentes anteriores. Dissertação (Graduação em Odontologia) - Faculdade de Odontologia de Araçatuba. Universidade Estadual Paulista Julia de Mesquita Filho, Araçatuba, 2016; 33 p

22. SILVA AM, et al. Avaliação da resistência de união de reparos em resina composta através do teste de cisalhamento. Revista da JOPIC, 2018; 1(2): 69-84

23. SILVA LA, et al. Reanatomização e recontorno cosmético de dentes anteriores com anomalia de forma: relato de caso clínico. Arq. Bras. Odontol. 2006; 1: 55-59

24. VIEIRA APSB, et al. Diferença de cor entre resinas compostas de lotes diferentes de acordo com a escala vita. Revista Campo do Saber, 2018; 4(5): 86-100. 